\title{
An algebraic approach to the Tavis-Cummings problem
}

\author{
I. P. Vadeiko 7 and G. P. Miroshnichenkof \\ Department of Mathematics, Saint-Petersburg Institute of Fine Mechanics and Optics, \\ 14 Sablinskaya str., St. Petersburg, 197101, Russia \\ A. V. Rybin f $^{\text {and J. Timonen }}$ \\ Department of Physics, University of Jyväskylä, PO BOX 35, FIN-40351, Jyväskylä, Finland.
}

(Dated: October 22, 2018)

\begin{abstract}
An algebraic method is introduced for an analytical solution of the eigenvalue problem of the Tavis-Cummings (TC) Hamiltonian, based on polynomially deformed $s u(2)$, i.e. $s u_{n}(2)$, algebras. In this method the eigenvalue problem is solved in terms of a specific perturbation theory, developed here up to third order. Generalization to the $\mathcal{N}$-atom case of the Rabi frequency and dressed states is also provided. A remarkable enhancement of spontaneous emission of $\mathcal{N}$ atoms in a resonator is found to result from collective effects.
\end{abstract}

PACS numbers: 42.50.-p

Keywords: polynomial deformation of $s u(2), \mathcal{N}$-atom micromaser, Tavis-Cummings Hamiltonian, a structure polynomial.

\section{INTRODUCTION}

We consider here the collective behavior of the system of $\mathcal{N}$ two-level atoms coupled to a single mode of the electromagnetic field in a resonator. The useful form for the atom-field interaction was proposed in the rotating wave approximation (RWA) by Tavis and Cummings [1]. In their model $\mathcal{N}$ identical two-level atoms interact via dipole coupling with a single-mode quantized radiation field at resonance, so that the Hamiltonian is given by

$$
\begin{gathered}
H=H_{0}+V, \quad H_{0}=\omega \cdot a^{\dagger} a+\omega_{0}\left(S_{3}+\frac{\mathcal{N}}{2}\right), \\
V=g\left(a^{\dagger} \cdot S_{-}+a \cdot S_{+}\right) .
\end{gathered}
$$

Here $\omega$ is a frequency of the electromagnetic field and $\omega_{0}$ is the level splitting of the two-level atoms. The operators $S_{3}, S_{ \pm}$are collective spin variables of $\mathcal{N}$ two-level atoms. These operators are defined as

$$
S_{3}=\sum_{j=1}^{\mathcal{N}} \sigma_{3}^{j}, S_{ \pm}=\sum_{j=1}^{\mathcal{N}} \sigma_{ \pm}^{j},
$$

where $\sigma$ 's are Pauli matrices. They satisfy the su(2) algebra. $a, a^{\dagger}$ are the annihilation and creation operators of the field. Due to historical reasons the Tavis-Cummings (TC) model is often called the Dicke model [2]. We concentrate here on the case of exact resonance, i.e. $\omega=\omega_{0}$. In this case the system exhibits a most interesting collective behavior. For simplicity, time will be measured in units of the coupling constant $g$, i.e. we assume in the following that $g=1$.

\footnotetext{
*Electronic address: vadeiko@mkk.ifmo.ru

${ }^{\dagger}$ Electronic address: mirosh@mkk.ifmo.ru

${ }^{\ddagger}$ Electronic address: cvbin@phvs.jvu.fi

$\S$ Electronic address: jussi.timonen@phys.jyu.fi
}

The one-atom version of the TC model, i.e. the JaynesCummings model(JC) [3] has recently attracted much attention because of e.g. the spectacular experiments reported in [4]. In this work the properties of a dilute flux of atoms excited to Rydberg states and interacting with a single quantum mode of the electromagnetic field were analyzed in a high-Q cavity. The results reported in [4] demonstrate that the physical assumptions underlying the JC model can indeed be experimentally realized. This triggered a lot of theoretical interest towards the one-atom micromaser(microlaser) (see [5, 6, 7] and references therein). The analysis of the JC model is greatly simplified by the fact that the eigenvalue problem of the Jaynes-Cummings Hamiltonian can be solved exactly. An algebraic approach to the solution of generalized Jaynes-Cummings types of models can be found in 8, 9. The approach to the Tavis-Cummings type models based on the Algebraic Bethe Ansatz is formulated in 10].

An analytical solution of the $\mathcal{N}$-atom case, i.e. the TC model, is much more complicated than that of JC model and is thus still far from completion. In this article we analyze this model and develop an analytical technique that is applicable to a variety of problems of TC type.

To begin with we notice that Hamiltonian (11) belongs to a class of operators that can be expressed in the form:

$$
H=f\left(A_{0}\right)+g \cdot\left(A_{+}+A_{-}\right) .
$$

Here $f(x)$ is an analytic function of $x$ with real coefficients, while the operators $A_{ \pm}$satisfy the commutation relations

$$
\left[A_{0}, A_{ \pm}\right]= \pm A_{ \pm}
$$

We also assume the operator $H$ to be self-adjoint. This means that, for any irreducible representation (irrep), the operators $A_{0}, A_{ \pm}$must satisfy the conditions

$$
\left(A_{0}\right)^{\dagger}=A_{0},\left(A_{-}\right)^{\dagger}=A_{+}
$$


It is worth mentioning that Hamiltonians of type (11) are also used in description of Raman and Brillouin scattering, and frequency conversion, as well as parametric amplification that involves trilinear boson operators 11:

$$
\mathrm{H}=\omega a^{\dagger} a+\sum_{i=1}^{2} \omega_{i} \cdot b_{i}^{\dagger} b_{i}+g\left(b_{1}^{\dagger} b_{2} a+b_{1} b_{2}^{\dagger} a^{\dagger}\right)
$$

The equivalence of Hamiltonians (11) and (6) can be readily seen by applying the Schwinger transformation

$$
S_{+}=b_{1}^{\dagger} b_{2}, S_{-}=b_{1} b_{2}^{\dagger}, S_{3}=\frac{1}{2}\left(b_{1}^{\dagger} b_{1}-b_{2}^{\dagger} b_{2}\right) .
$$

The Hamiltonians of the type of Eq. (3) are usually analyzed by approximating them with an exactly solvable one. The solvable Hamiltonian is usually quadratic in the boson operators or linear in the generators of a classical Lie algebra. In this approach the so-called parametric approximation or its variations [12, 13, 14] are often used. The basic assumption of this approximation is that one of the quantum modes or subsystems is prepared in a highly excited (often coherent) state, or in a state close to the vacuum. This approach, however, puts certain restrictions on the type of possible initial conditions as well as on the time span over which the quantum dynamics can be followed. Another type of an approach to the solution of Hamiltonian (3) is based on perturbation theory for nonlinear algebras 15]. This approach requires however the existence of a small parameter. In the TC model such a parameter was found in the case the atoms are in completely symmetric states $\left(r=\frac{\mathcal{N}}{2}\right)$ [16, 17.

The basic idea of the present work is to combine these two approaches through algebraic methods as applied e.g. in $18,19,20,21)$. To this end we reformulate the Hamiltonian in terms of an algebra that better allows the diagonalization of the Hamiltonian. This idea was already used by Holstein and Primakoff [22]. They expressed the generators $S_{3}, S_{ \pm}$of the su(2) algebra in terms of boson operators $b, b^{\dagger}$,

$$
S_{3}=r-b^{\dagger} b, S_{+}=\sqrt{2 r} \sqrt{1-\frac{b^{\dagger} b}{2 r}} b, S_{-}=\left(S_{+}\right)^{\dagger} .
$$

Here $r$ is an index that characterizes the irrep of $s u(2)$. However, in 22 the square root in the transformation Eq. (8) was in the end replaced by unity, which amounts to applying the so-called "weak field" approximation $\left(\left\langle b^{\dagger} b\right\rangle \ll 2 r\right)$. Obviously, this approximation corresponds to zeroth order in the expansion of the problem with respect to parameter $\frac{1}{2 r}$. Transfromation (8) has also been applied [23] with expansion up to second order.

We consider here the case when the operators $A_{0}, A_{ \pm}$ in Eq. (3) are generators of a polynomial deformation $s u_{n}(2)$ of the Lie algebra $s u(2)$ 21, 24, 25]. Numerous physical applications exist for polynomially deformed algebras 22, 26, 28, 29, 30, 31, 32, 33, 34, 35, 36, 37.
A particularly interesting, in view of the present problem, application of deformed algebras was developed by Karassiov (see in [26] and refs. therein). The method to be introduced below is an extension of Karassiov's method.

We introduce here the notion of a Polynomial Algebra of Excitations (PAE). In this algebra the coefficients of the structure polynomials are $c$-numbers, rather than Casimir operators as is typical of polynomial deformations. We derive an exact mapping between isomorphic representations of two arbitrary PAE. We also provide a classification of isomorphic representations of Polynomial Algebras of Excitations. In our approach classes of isomorphic representations are specified by the multiplicity of the maximal and minimal eigenvalues of the operator $A_{0}$ in the given representation. We formulate then an analytical approach that allows us to expand the Hamiltonian, when expressed in terms of PAE, as a perturbation series.

For completely symmetric states of atoms our results agree with those reported in 16, 17]. Our formalism provides however a solution of the problem for any value of $r$, which allows us to discuss new physical effects in the Dicke model.

The article is organized as follows. In the next Section II we discuss the irreducible representations of PAE. In the Section III we apply the general approach to the Tavis-Cummings model, and in Section IV construct the perturbation theory for the TC Hamiltonian and solve its eigenvalue problem up to third order. The generalized $\mathcal{N}$-atom quantum Rabi frequency is defined for arbitrary quantum states of the system. In the Section V we use the zero order approximation for the TC Hamiltonian to calculate the intensity of spontaneous emission of atoms prepared in the state of thermal equilibrium with the resonator mode. We show that the correlation of the atoms due to interaction with the field gives rise to the enhancement of spontaneous emission as compared to the atoms in the absence of resonator. In conclusion, we discuss possible further applications of the methods developed here. Technical details of the algebraic manipulations are given in the Appendix.

\section{IRREDUCIBLE REPRESENTATIONS OF THE POLYNOMIAL ALGEBRA OF COLLECTIVE EXCITATIONS}

The coefficients of the structure polynomial of a polynomially deformed algebra are usually expressed through the Casimir operators of the algebra. In this Section we discuss representations of a special class (PAE) of polynomially deformed algebras when the coefficients of the structure polynomial are $c$-numbers. We denote a PAE with a structure polynomial of order $\kappa$ as $\mathfrak{U}_{\kappa}$. Formally $\mathfrak{U}_{\kappa}$ is an associative algebra with unity, defined by three generators $A_{ \pm}, A_{0}$. These generators satisfy two basic commutation relations, Eq. (4). As can be readily seen 
from these commutation relations, $\left[A_{0}, A_{+} A_{-}\right]=0$. We can thus assume that

$$
A_{+} A_{-}=p_{\kappa}\left(A_{0}\right)=c_{0} \prod_{i=1}^{\kappa}\left(A_{0}-q_{i}\right) .
$$

Here $p_{\kappa}(x)$ is a structure polynomial of order $\kappa$, whose coefficients are generally complex numbers. The terminology is chosen in analogy to the structure functions of quantum algebras (q-deformed algebras) [27], and the structure constants of the linear Lie algebras. The set of $\kappa$ real roots of the structure polynomial is denoted as $\left\{q_{i}\right\}_{i=1}^{\kappa}$. In physical applications the operators $A_{ \pm}$of Eq. (4) often play the role of creation and annihilation operators of collective excitations. Therefore, hereafter the algebra $\mathfrak{U}_{\kappa}$ will be referred to as the polynomial algebra of excitations (PAE) of order $\kappa$. Notice that this algebra is different from the algebra $s u_{n}(2)$. All PAE's are completely defined by $\kappa+1$ c-numbers, the coefficient $c_{0}$ and the $\kappa$ roots $\left\{q_{i}\right\}$ of the structure polynomial. In the case of $s u_{n}(2)$, however, the structure polynomial has some coefficients in the form of Casimir operators.

Below we consider two elementary, but important for the following, examples of PAE. To begin we assume, without loss of generality, that $c_{0}= \pm 1$. Indeed, in the case $\left|c_{0}\right| \neq 1$, it is always possible to renormalize the generators of $\mathfrak{U}_{\kappa}$,

$$
A_{ \pm} \rightarrow\left|c_{0}\right|^{-\frac{1}{2}} A_{ \pm}
$$

such that the commutation relations Eq. (4) remain intact. Using these commutation relations it can also be readily seen that

$$
A_{0} A_{ \pm}=A_{ \pm}\left(A_{0} \pm 1\right), A_{-} A_{+}=p_{\kappa}\left(A_{0}+1\right) .
$$

As indicated above, $A_{+}$and $A_{-}$have the physical meaning of creation and annihilation operators of collective excitations (quasiparticles), while $A_{0}$ is the operator for the number of excitations. The most simple and important example of a PAE of first order, $\mathfrak{U}_{1}$, is provided by the well-known Heisenberg-Weil Lie algebra, viz.

$$
\begin{gathered}
b^{\dagger} \rightarrow A_{+}, b \rightarrow A_{-}, b^{\dagger} b \rightarrow A_{0}, \\
c_{0}=1, q_{1}=0 .
\end{gathered}
$$

Here $b, b^{\dagger}$ are the usual boson operators. For the sake of simplicity, in what follows we will denote the generators of $\mathfrak{U}_{1}$ by $b, b^{\dagger}$. The algebra $\mathfrak{U}_{1}$ allows us to construct the irrep of any other PAE of higher order $\kappa>1$ as a multiple tensor product of $\mathfrak{U}_{1}$.
As practical example let us describe in detail a second order PAE denoted in the paper by $\mathbb{S}_{r}$. It is relevant to the algebra $s u(2)$ given by the commutation relations

$$
\left[S_{3}, S_{ \pm}\right]= \pm S_{ \pm}, \quad\left[S_{+}, S_{-}\right]=2 S_{3}
$$

It is plain that

$$
S_{+} S_{-}=S^{2}-S_{3}^{2}+S_{3}
$$

For every irrep the Casimir operator $S^{2}$ is equal to $r(r+1) I$, where $I$ is the identity operator. The corresponding $\mathrm{PAE}$ of second order, $\mathbb{S}_{r}$, is constructed such that

$$
\begin{gathered}
S_{+} \rightarrow A_{+}, S_{-} \rightarrow A_{-}, S_{3} \rightarrow A_{0} ; \\
c_{0}=-1, q_{1}=-r, q_{2}=r+1 .
\end{gathered}
$$

Obviously, $\mathbb{S}_{r}$ has a matrix irrep isomorphic to the irrep of su(2) with the same $r$. Notice that only in this representation of $\mathbb{S}_{r}$ the condition Eq.(5) is fulfilled. This gives us a motivation to denote the three generators of $\mathbb{S}_{r}$ as $\tilde{S}_{3}, \tilde{S_{ \pm}}$.

Below we describe a general method to construct a realization of any PAE $\mathfrak{U}_{\kappa}$ through the algebra $\mathfrak{U}_{1}$. Choosing a root $q_{j}$ of the structure polynomial we can construct this realization in the form

$$
\begin{gathered}
A_{0}=b_{j}^{\dagger} b_{j}+q_{j}, A_{+}=\sqrt{c_{0} \prod_{i=1, i \neq j}^{n}\left(b_{j}^{\dagger} b_{j}+q_{j}-q_{i}\right)} b_{j}^{\dagger}, \\
A_{-}=b_{j} \sqrt{c_{0} \prod_{i=1, i \neq j}^{n}\left(b_{j}^{\dagger} b_{j}+q_{j}-q_{i}\right)} .
\end{gathered}
$$

Here $b_{j}, b_{j}^{\dagger}$ are the usual boson operators associated with the chosen root $q_{j}$ of the structure polynomial $p_{\kappa}(x)$. The corresponding Fock vectors, i.e. the eigenvectors of $b_{j}^{\dagger} b_{j}$, are denoted as $|n\rangle_{j}$. In what follows the chosen root $q_{j}$ of the structure polynomial will be referred to as the pivotal root. Notice that the product in $(16)$ always contains exactly $\kappa$ multipliers regardless of the multiplicity of the root. Since $b_{j}^{\dagger} b_{j}$ has a well-defined discrete spectrum, the square root function is defined in the form of spectral decomposition. We specify the branch of the square root by choosing $\sqrt{-1}=i$. It is easy to see that the condition Eq.(9) and the commutation relations Eq.(4) for generators of $\mathfrak{U}_{\kappa}$ are satisfied.

A useful automorphism $\hat{T}$,

$$
\hat{T} b^{\dagger}=i \cdot b, \hat{T} b=i \cdot b^{\dagger} \Rightarrow \hat{T} b^{\dagger} b=-\left(b^{\dagger} b+1\right),
$$

of $\mathfrak{U}_{1}$ allows us to construct another realization of $\mathfrak{U}_{\kappa}$ through $\mathfrak{U}_{1}$, 


$$
A_{0}=q_{j}-1-b_{j}^{\dagger} b_{j}, \quad A_{+}=b_{j} \sqrt{\left(-c_{0}\right) \prod_{i=1, i \neq j}^{\kappa}\left(q_{j}-q_{i}-b_{j}^{\dagger} b_{j}\right)}, \quad A_{-}=\sqrt{\left(-c_{0}\right) \prod_{i=1, i \neq j}^{\kappa}\left(q_{j}-q_{i}-b_{j}^{\dagger} b_{j}\right)} b_{j}^{\dagger} .
$$

It is worth mentioning that transformations similar to Eqs. (16), (18) have been introduced earlier under the name of multiboson realizations of Bose operators. These multiboson realizations satisfy the usual boson commutation relations, $\left[A, A^{\dagger}\right]=1$ [28, 29, 30].

Applying the realizations Eqs.(16),(18) in any representation of $\mathfrak{U}_{1}$, we can construct a representation of $\mathfrak{U}_{\kappa}$. In the case of realization Eq.(16), an irreducible representation of $\mathfrak{U}_{\kappa}$ is constructed through application of the operator $A_{+}$to the vacuum vector $|0\rangle_{j}$. The finite dimensional representation can be constructed in the case when the root $q_{j+1}$ is separated from $q_{j}$ by a natural number $d$. Then it can be readily seen that $A_{+}^{d}|0\rangle_{j}=0$. Indeed, provided that $A_{-}=A_{+}^{\dagger}$, it is not difficult to show that the norm of the vector $A_{+}^{d}|0\rangle_{j}$ vanishes, i.e. ${ }_{j}\left\langle 0\left|A_{-}^{d} A_{+}^{d}\right| 0\right\rangle_{j}=0$. The general construction can be exemplified by $\mathbb{S}_{r}$. When $r$ is integer or half-integer, $d=q_{1}-q_{2}=2 r+1$ is integer, and $\mathbb{S}_{r}$ has a finite dimensional irrep which is isomorphic to the corresponding irrep of $s u(2)$.

In the case of realization Eq.(18), the corresponding irrep is constructed through application of the operator $A_{-}$to the vacuum vector $|0\rangle_{j}$. Using an argumentation similar to that given above, it can be seen that the finite dimensional representation can now be constructed provided that the root $q_{j-1}$ is separated from $q_{j}$ by a natural number.

The meaning of transformations Eqs. (16) and (18) becomes now transparent . The realization Eq.(16) corresponds to the case when the operator $A_{+}$is a creation operator, while the realization Eq.(18) corresponds to the case when $A_{+}$is an annihilation operator.

Since the spectrum of operator $b_{j}^{\dagger} b_{j}$ is a set of natural numbers and zero, the operator $A_{0}-q_{j}$ in Eq. (16) has a non-negative spectrum. Therefore, the argument of the square root function is a positive operator in the finite dimensional subspace, where the structure polynomial $p_{\kappa}\left(A_{0}\right)$ has non-negative spectrum. In this case the operators $A_{+}$and $A_{-}$are Hermitian conjugated. In the subspace corresponding to the negative values of the spectrum of the structure polynomial, the argument of the square root function in Eq.(16) has a negative spectrum. The operators will be anti-conjugated, i.e. $\left(A_{-}\right)^{\dagger}=-A_{+}$, which is not plausible. Thus, in a physical problem (see (5)), we should only consider those irrep for which the spectrum of $p_{\kappa}\left(A_{0}\right)$ is nonnegative. The same is true for Eq. (18).

The two relations, Eqs.(16) and (18), between any two algebras $\mathfrak{U}_{\kappa}$ and $\mathfrak{U}_{1}$, show that there is no principal difference as to how exactly the meanings of the creation and annihilation operators of collective excitations are prescribed to the pair $A_{ \pm}$. The important point is that the pair exists. It is the physical problem in question which prescribes the meaning of operators $A_{ \pm}$and determines the location of the equidistant spectrum of $A_{0}$ on the real axis. Should one be interested in the eigenvalues of $A_{0}$, to the right of the pivotal root $q_{j}$, it is necessary to choose the transformation Eq.(16), while for the region to the left of $q_{j}$, it is necessary to use the realization (18). As was explained above, we choose the region such that the structure polynomial is nonnegative.

The general considerations given above can be illustrated by the $\mathbb{S}_{r}$ algebra. In the case of the realization of $\mathbb{S}_{r}$ connected to the pivotal root $q_{1}=-r$ it is necessary to use Eq.(16). This leads to the conventional HolsteinPrimakoff representation,

$$
\tilde{S}_{3}=b_{1}^{\dagger} b_{1}-r, \tilde{S}_{+}=\sqrt{2 r+1-b_{1}^{\dagger} b_{1}} b_{1}^{\dagger}, \tilde{S}_{-}=\left(\tilde{S}_{+}\right)^{\dagger} .(19)
$$

In the case of $q_{2}=r+1$, we use the realization Eq. (18),

$$
\tilde{S}_{3}=r-b_{2}^{\dagger} b_{2}, \tilde{S}_{+}=b_{2} \sqrt{2 r+1-b_{2}^{\dagger} b_{2}}, \tilde{S}_{-}=\left(\tilde{S}_{+}\right)^{\dagger} .
$$

The spectra of $b_{1}^{\dagger} b_{1}$ and $b_{2}^{\dagger} b_{2}$ are limited from above by the value $2 r$, while the subspace spanned by eigenvectors $|n\rangle_{1,2}(n=0,1, \ldots 2 r)$ of operator $A_{0}$ is irreducible (cf. Eq. (8)).

Obviously all the realizations of PAE constructed through Eqs. (16), (18) are fully characterized by the dimension $d$ of the invariant subspace and by the order $k_{-}$of the left and $k_{+}$of the right roots defining the corresponding irreducible representation. Thus an irrep of PAE is characterized by a set of parameters $\left\{k_{-}, k_{+}, d\right\}$. Such irrep we will denote by $R\left(k_{-}, k_{+}, d\right)$. For instance, $R(1,0, \infty)$ means the representation of $\mathfrak{U}_{1}$, while the irrep of $\mathbb{S}_{r}$ is $R(1,1,2 r+1)$.

An isomorphism between irreps of $\mathfrak{U}_{\kappa}$ and $\mathfrak{U}_{\kappa^{\prime}}$ that belong to the same class $R\left(k_{-}, k_{+}, d\right)$, is given by

$$
\begin{gathered}
A_{0}=A_{0}^{\prime}+\left(q_{j}-q_{j^{\prime}}^{\prime}\right), \\
A_{+}=\sqrt{\frac{c_{0} \prod_{i=1}^{\kappa}\left(A_{0}^{\prime}+q_{j}-q_{j^{\prime}}^{\prime}-q_{i}\right)}{c_{0}^{\prime} \prod_{i^{\prime}=1}^{k^{\prime}}\left(A_{0}^{\prime}-q_{i^{\prime}}^{\prime}\right)} A_{+}^{\prime},} \\
A_{-}=A_{-}^{\prime} \sqrt{\frac{c_{0} \prod_{i=1}^{\kappa}\left(A_{0}^{\prime}+q_{j}-q_{j^{\prime}}^{\prime}-q_{i}\right)}{c_{0}^{\prime} \prod_{i^{\prime}=1}^{k^{\prime}}\left(A_{0}^{\prime}-q_{i^{\prime}}^{\prime}\right)}},
\end{gathered}
$$


or by

$$
\begin{gathered}
A_{0}=\left(q_{j^{\prime}}^{\prime}+q_{j}-1\right)-A_{0}^{\prime}, \\
A_{+}=A_{-}^{\prime} \sqrt{\frac{c_{0} \prod_{i=1}^{K}\left(q_{j^{\prime}}^{\prime}+q_{j}-A_{0}^{\prime}-q_{i}\right)}{c_{0}^{\prime} \prod_{i^{\prime}=1}^{\kappa^{\prime}}\left(A_{0}^{\prime}-q_{i^{\prime}}^{\prime}\right)}}, \\
A_{-}=\sqrt{\frac{c_{0} \prod_{i=1}^{\kappa}\left(q_{j^{\prime}}^{\prime}+q_{j}-A_{0}^{\prime}-q_{i}\right)}{c_{0}^{\prime} \prod_{i^{\prime}=1}^{\kappa^{\prime}}\left(A_{0}^{\prime}-q_{i^{\prime}}^{\prime}\right)}} A_{+}^{\prime} .
\end{gathered}
$$

In Eqs. (21),(22) the operator argument of the square root function should be taken after identical multipliers in the nominator and denominator are cancelled. The pivotal roots $q_{j}$ and $q_{j^{\prime}}^{\prime}$ define a vacuum vector of the irrep. Compare these expressions with Eqs.(16),(18).

To recapitulate: in the general case two irreducible representations $R\left(k_{-}, k_{+}, d\right)$ and $R^{\prime}\left(k_{-}^{\prime}, k_{+}^{\prime}, d^{\prime}\right)$ of $\mathfrak{U}_{\kappa}$ and $\mathfrak{U}^{\prime}{ }_{\kappa^{\prime}}$ are isomorphic provided that $d=d^{\prime}$, and

$$
k_{-}=k_{-}^{\prime}, k_{+}=k_{+}^{\prime} \text { or } k_{-}=k_{+}^{\prime}, k_{+}=k_{-}^{\prime} .
$$

For symmetric irreps, i.e. when $k_{+}=k_{-}$, these conditions coincide. The isomorphism is a consequence of the fact that, under conditions (23), one can choose $q_{j}\left(q_{j}^{\prime}\right)$ in such a way that the functions under the square root in Eqs.(21), (22) do not have zeros in the spectrum of operator $A_{0}$, and therefore we can consider the square root as a single-valued analytic function. This means that transformations are invertible, analytic, and therefore define an isomorphism of irreps.

The constructed transformations give us a tool to realize $\mathfrak{U}_{\kappa}$ in terms of a simpler PAE with the same type of irrep. This procedure will be applied below to the Tavis-Cummings Hamiltonian.

\section{THE TAVIS-CUMMINGS HAMILTONIAN IN TERMS OF THIRD-ORDER PAE}

The interaction part of the Hamiltonian Eq. (11) can be expressed in terms of third-order PAE. The generators $M_{0}, M_{ \pm}$of this algebra are realized as

$$
M_{-}=a \cdot S_{+}, M_{+}=a^{\dagger} \cdot S_{-}, M_{0}=\frac{a^{\dagger} a-S_{3}}{2} .
$$

It is plain that these generators satisfy the commutation relations Eqs. (4). The generators of the algebra $M_{0}, M_{ \pm}$ commute with the operators

$$
M=a^{\dagger} a+S_{3}+r, S^{2}=S_{3}^{2}+\frac{1}{2}\left(S_{+} S_{-}+S_{-} S_{+}\right) .
$$

Hereafter we use the same notation $M$ both for the Casimir operator and its eigenvalue, if no confusion arises. We show below that the eigenvalues $M, r(r+1)$ of

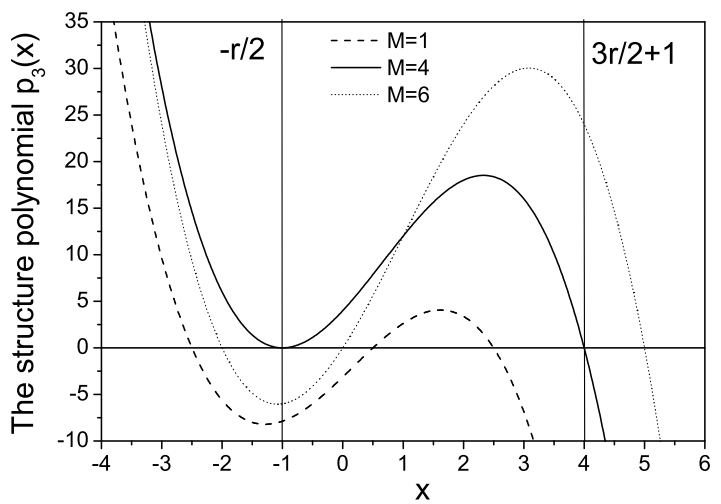

FIG. 1: The structure polynomial $p_{3}(x)$ of the $\mathbb{M}_{M, r}$ algebra for $r=2$.

the operators of Eq. (25) parameterize the PAE in question. We thus denote this PAE as $\mathbb{M}_{M, r}$. The structure polynomial of $\mathbb{M}_{M, r}$ can be expressed in the form

$$
\begin{gathered}
p_{3}\left(M_{0}\right)=M_{+} M_{-}=a^{\dagger} a\left(S^{2}-S_{3}^{2}-S_{3}\right)= \\
a^{\dagger} a\left(r-S_{3}\right)\left(r+S_{3}+1\right)= \\
-\left(M_{0}+\frac{M-r}{2}\right)\left(M_{0}-\frac{M-3 r}{2}\right)\left(M_{0}-\frac{M+r+2}{2}\right) .
\end{gathered}
$$

The parameters of this structure polynomial are

$$
\begin{gathered}
c_{0}=-1, q_{1}=-\frac{M-r}{2}, \\
q_{2}=\frac{M-r}{2}-r, q_{3}=\frac{M-r}{2}+r+1,
\end{gathered}
$$

and its behavior as a function of $M_{0}$ is given in Fig. 1 .

We turn next to the description of finite dimensional irrep of $\mathbb{M}_{M, r}$. In physical applications the parameter $r$ has the meaning of collective Dicke index. This index runs from $\varepsilon(\mathcal{N})=\frac{1-(-1)^{\mathcal{N}}}{4}$ to $\frac{\mathcal{N}}{2}$ with unit steps, while $M$ can be any natural number including zero. Thus $q_{3}$ is the biggest positive root of first order. If $M<2 r$, then $q_{1}>q_{2}$; if $M>2 r$ then $q_{1}<q_{2}$; the case $M=2 r \Rightarrow$ $q_{1}=q_{2}$ corresponds to a root of second order. A typical plot of the structure polynomial is shown in Fig. 1 for these three cases. A finite dimensional representation of $\mathbb{M}_{M, r}$ (where $\left(M_{+}\right)^{\dagger}=M_{-}$) corresponds to the positive spectrum of $p_{3}\left(M_{0}\right)$. The spectrum is limited from the right by $q_{3}$ and from the left by $q_{1}$ or $q_{2}$. Notice that the number $M$ is conserved. Therefore this number is determined by the initial state. The different values of $M$ and $r$ define different algebras $\mathbb{M}_{M, r}$, whose single physical finite dimensional representation we will call a zone. The case $M<2 r$ corresponds to nearby zones. The two largest roots are $q_{1}$ and $q_{3}$ and the irrep has the type $R(1,1, M+1)$. Consequently, the well-known weak-field limit corresponds to nearby zones.

The case $M>2 r$ corresponds to remote zones. The two largest roots are $q_{2}$ and $q_{3}$, and the corresponding 


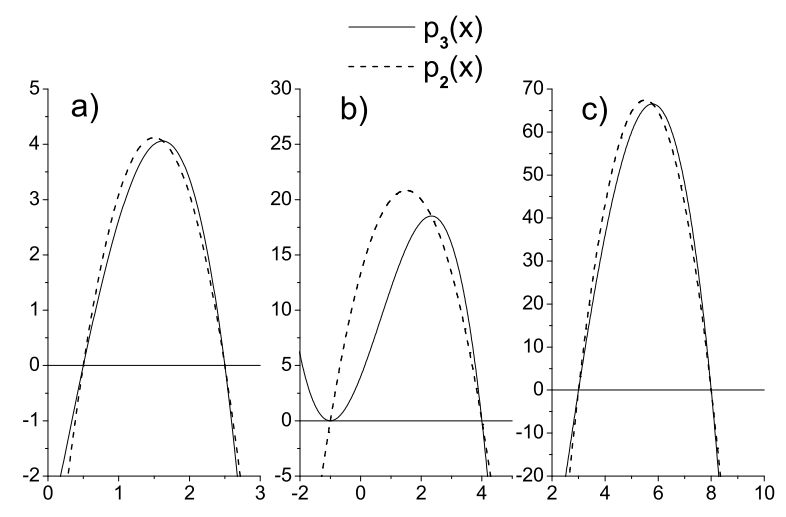

FIG. 2: Approximation of the structure polynomial $p_{3}(x)$ by the parabolic $p_{2}(x)$ for $r=2$. a) $M=1$; b) $M=4$; c) $M=12$.

irrep is of the type $R(1,1,2 r+1)$. Notice that the region $M>>2 r$ is usually called the strong-field limit.

In the special case $2 r=M$, referred to intermediate zone, the algebra $\mathbb{M}_{M, r}$ possesses an irrep of the type $R(2,1,2 r+1)$. It is the only irreducible representation that principally differs from all the others.

As indicated above, the simplest PAE with irrep of the type $R\left(1,1, d\right.$ ) is $\mathbb{S}_{\tilde{r}}$ (we use here $\tilde{r}$ to distinguish it from the (physical) collective index $r$ ). It would be convenient to solve the eigenvalue problem in terms of the simplest algebra $\mathbb{S}_{\tilde{r}}$. Notice that in any finite dimensional irrep of PAE characterized by $R(1,1, d)$, the structure polynomial of the algebra can be approximated by a parabolic curve. This is shown in Fig. 2 (for $M \neq 2 r$ ) for the structure polynomial of $\mathbb{M}_{M, r}$. The larger(smaller) $M$ is in comparison with $2 r$, the better is the approximation. However, for $M \approx 2 r$ the approximation is not satisfactory (see Fig. 2 $2 \mathrm{~b}$ ). In the regions where the approximation is adequate, it is then not difficult to diagonalize the operator $V=S_{+}+S_{-}$, defined in terms of generators of the conventional su(2) algebra. The latter has a parabolic structure polynomial. In Fig. 2 the two roots of $p_{2}(x)$ are chosen to be equal to the corresponding two roots of $p_{3}(x)$. The choice of $c_{0}$ in $p_{2}(x)$ will be explained below. Thus in the cases of nearby or remote zones, it is convenient to study the problem in terms of algebra $\mathbb{S}_{\tilde{r}}$. The approximation illustrated in Fig. 22 indicates that the TC problem can be solved via an appropriate perturbation theory.

To begin with we consider the transformation of $\mathbb{M}_{M, r}$ to $\mathbb{S}_{\tilde{r}}$ for the case of remote zones. The dimension of a remote zone is $2 r+1$, and the algebra $\mathbb{S}_{\tilde{r}}$ should be characterized by $\tilde{r}=r$. The finite dimensional irrep of $\mathbb{S}_{\tilde{r}}$ is isomorphic to the corresponding irreducible representation of the atomic subsystem. For the pivotal root $q_{j}$ we choose the largest root that bounds the irrep of $\mathbb{M}_{M, r}$ from the right (the $q_{3}$ in (27)), while as the root $q_{j^{\prime}}^{\prime}$ we take the $q_{1}$ of $\mathbb{S}_{r}$ from Eq. (15). Applying the mapping
(22) we obtain

$$
\begin{gathered}
M_{0}=\frac{M-r}{2}-\tilde{S}_{3}, M_{+}=\tilde{S}_{-} \sqrt{\left(M-r+1-\tilde{S}_{3}\right)}, \\
M_{-}=\sqrt{\left(M-r+1-\tilde{S}_{3}\right)} \tilde{S}_{+}
\end{gathered}
$$

The spectrum $\{\tilde{m}\}$ of the operator $\tilde{S}_{3}$ belongs to the region $-r \leq \tilde{m} \leq r$, consequently the argument of the square root function in Eq. (28) is positive in the remote zones $(M-r>r)$. The relations Eqs.(28) express the generators of algebra $\mathbb{M}_{M, r}$ as analytic function of the generators of the $\mathbb{S}_{r}$ algebra. they thus allow us to approximate the more complex algebra $\mathbb{M}_{M, r}$ of third order by a simpler algebra of second order.

Before we begin to study this approximation we construct a realization of $\mathbb{S}_{r}$ in terms of boson and spin variables. ¿From Eqs.(24),(25), and (28) it follows that in remote zones

$$
\tilde{S}_{3}=S_{3}, \tilde{S}_{+}=\frac{1}{\sqrt{a^{\dagger} a+1}} a \cdot S_{+}, \quad \tilde{S}_{-}=\left(\tilde{S}_{+}\right)^{\dagger} .
$$

Notice that the subspaces that correspond to remote zones do not contain the vacuum state of the field. It is also worth mentioning that the matrix representation of operator $\frac{1}{\sqrt{a^{\dagger} a+1}} a$ is $\delta_{n, n+1}$ in any remote zone. This operator has been considered before as a earlier in terms of phase operator 11, 18, 19].

We turn now to the nearby zones $M<2 r$. For this region the mapping of the algebra $\mathbb{M}_{M, r}$ to the algebra $\mathbb{S}_{\tilde{r}}$ is realized through procedure similar to that described above for remote zones. Notice that the dimension of nearby zones is $d=q_{3}-q_{1}=M+1$, and therefore $\tilde{r}=\frac{M}{2}$ (cf. Fig. 2a). For the nearby zones there is no simple correspondence to a physical representation like that of Eq.(29). The particular choice of the pivotal root $q_{j^{\prime}}^{\prime}$ is unimportant and we use the same choice as before. Applying Eq.(22) we obtain

$$
\begin{gathered}
M_{0}=\frac{r}{2}-\tilde{S}_{3}, M_{+}=\tilde{S}_{-} \sqrt{\left(\frac{4 r-M}{2}+1-\tilde{S}_{3}\right)}, \\
M_{+}=\left(M_{-}\right)^{\dagger} .
\end{gathered}
$$

Since all the eigenvalues of the operator $\tilde{S}_{3}$ belong to the interval $-\tilde{r}$ to $\tilde{r}$, the argument of the square root function does not have zero eigenvalues in the nearby zones. The realization of $\mathbb{S}_{\tilde{r}}$ through spin and boson variables is then given by

$$
\begin{array}{r}
\tilde{S}_{3}=\frac{M}{2}-a^{\dagger} a, \tilde{S}_{+}=\frac{1}{\sqrt{r+1-S_{3}}} S_{+} a \\
\tilde{S}_{-}=S_{-} \frac{1}{\sqrt{r+1-S_{3}}} a^{\dagger} .
\end{array}
$$

Notice that the nearby zones do not contain the eigenvector $|r, r\rangle$ of $S_{3}$. 
To clarify the structure of intermediate zone we choose as the pivotal root as the third root of $p_{3}(x)$ of Eq. 27., and apply the transformation (18). We thus obtain

$$
\begin{gathered}
M_{0}=q_{3}-b_{3}^{\dagger} b_{3}-1=\frac{M+r}{2}-b_{3}^{\dagger} b_{3}, \\
M_{+}=b_{3} \sqrt{\left(M+1-b_{3}^{\dagger} b_{3}\right)\left(2 r+1-b_{3}^{\dagger} b_{3}\right)} \\
M_{-}=\sqrt{\left(M+1-b_{3}^{\dagger} b_{3}\right)\left(2 r+1-b_{3}^{\dagger} b_{3}\right)} b_{3}^{\dagger} .
\end{gathered}
$$

Here $b_{3}, b_{3}^{\dagger}$ are generators of the algebra $\mathfrak{U}_{1}$.

In the region $M>2 r$ the multiplier $\left(2 r+1-b_{3}^{\dagger} b_{3}\right)$ in Eq.(32) vanishes first. This corresponds to remote zones. In the region $M<2 r$ the multiplier that vanishes first is $\left(M+1-b_{3}^{\dagger} b_{3}\right)$. In the intermediate zone $M=2 r$ we have

$$
M_{+}=b_{3}\left(2 r+1-b_{3}^{\dagger} b_{3}\right), M_{-}=\left(2 r+1-b_{3}^{\dagger} b_{3}\right) b_{3}^{\dagger} .
$$

In the intermediate zone we thus obtain a special realization of the TC Hamiltonian,

$$
\begin{gathered}
H_{\{M=2 r\}}=\left(2 r+\frac{1}{2}\right)\left(b_{3}+b_{3}^{\dagger}\right)- \\
\frac{1}{2}\left[b_{3}^{\dagger} b_{3}\left(b_{3}+b_{3}^{\dagger}\right)+\left(b_{3}+b_{3}^{\dagger}\right) b_{3}^{\dagger} b_{3}\right] .
\end{gathered}
$$

The domain of the quantum space for this Hamiltonian is specified by the condition $n_{3} \leq 2 r$.

\section{DIAGONALIZATION OF THE TAVIS-CUMMINGS HAMILTONIAN}

Let us introduce an $\mathcal{N}$-atom generalization for arbitrary values $r$ and $M$ of the well-known quantum Rabi frequency such that

$$
\Omega_{R} \equiv\left\{\begin{array}{c}
2 \sqrt{M-r+\frac{1}{2}}, M \geq 2 r \\
2 \sqrt{\frac{4 r-M+1}{2}}, \quad M<2 r
\end{array}\right.
$$

For $r=\frac{\mathcal{N}}{2}$ our definition agrees with that used in 16 , 17]. Introducing a small parameter $\alpha \equiv\left(\frac{1}{2} \Omega_{R}\right)^{-2}$ we can rewrite the realizations of $M_{ \pm}$in nearby and remote zones (see Eqs. 28),(30)) in the form

$$
M_{+}=\frac{\Omega_{R}}{2} \tilde{S}_{-} \sqrt{1-\alpha\left(\tilde{S}_{3}-\frac{1}{2}\right)}, M_{-}=M_{+}^{\dagger}
$$

The diagonalization problem for the operator Eq.(11) can now be solved in each zone by means of perturbation theory with respect to the small parameter $\alpha$. One can show that the eigenvalues of the argument of the square root function in Eq.(36) are less then unity. Hence we can expand the square with respect to $\alpha$, and find thereby for the interaction part of the Hamiltonian

$$
\mathrm{V}=\frac{\Omega_{R}}{2}\left[\begin{array}{l}
\left.\left(\tilde{S}_{+}+\tilde{S}_{-}\right)-\frac{\alpha}{2}\left(\left(\tilde{S}_{3}-\frac{1}{2}\right) \tilde{S}_{+}+\tilde{S}_{-}\left(\tilde{S}_{3}-\frac{1}{2}\right)\right)-\frac{1}{2 !}\left(\frac{\alpha}{2}\right)^{2}\left(\left(\tilde{S}_{3}-\frac{1}{2}\right)^{2} \tilde{S}_{+}+\tilde{S}_{-}\left(\tilde{S}_{3}-\frac{1}{2}\right)^{2}\right)-{ }^{n}\right] \\
\left(\sum_{n=3}^{\infty} \frac{(2 n-3) ! !}{n !}\left(\frac{\alpha\left(\tilde{S}_{3}-\frac{1}{2}\right)}{2}\right)^{n} \tilde{S}_{+}+\tilde{S}_{-} \sum_{n=3}^{\infty} \frac{(2 n-3) ! !}{n !}\left(\frac{\alpha\left(\tilde{S}_{3}-\frac{1}{2}\right)}{2}\right)^{n}\right)
\end{array}\right]
$$

In the interaction representation the Hamiltonian coincides with $V$, we only need to diagonalize the latter. Up to third order in $\alpha$ we find that

$$
V=\frac{\Omega_{R}}{2}\left(\mathrm{~V}^{(0)}+\mathrm{V}^{(1)}+\mathrm{V}^{(2)}+\mathrm{V}^{(3)}\right),
$$

where the $V^{(n)}$ are terms of $n$-th order in $\alpha$, and are given in the Appendix A. In the Appendix we also show that unitary transformations $\tilde{U}_{k}, \quad k=0,1,2,3$, which bring the interaction operator into diagonal form:

$$
\bar{V} \equiv \tilde{U} \cdot V \cdot \tilde{U}^{-1}=\Omega_{R} \cdot \tilde{S}_{3}\left\{1+\left(\frac{\alpha}{4}\right)^{2} \cdot\left[5 \tilde{S}_{3}^{2}-3 \tilde{r}(\tilde{r}+1)+1\right]\right\}, \quad \tilde{U} \equiv \tilde{U}_{3} \tilde{U}_{2} \tilde{U}_{1} \tilde{U}_{0}
$$

The spectrum of the operator $V$ as given by Eq. 38) agrees with the results of 16, 17] for the symmetric states of the atoms.
We compared the third order solution Eq. (38) with the exact numerical diagonalization of $V$ and found that the result (38) is very accurate, especially for increasing values of $|\bar{M}-2 r|$. The results of this comparison are 


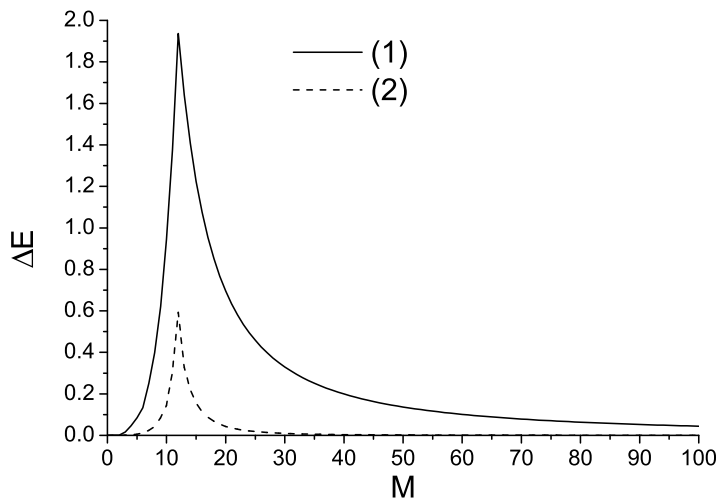

FIG. 3: Deviation of the eigenvalues of $V$ from their numerical values in zero (solid line) and second (dashed line) order in $\alpha$, for $r=6$.

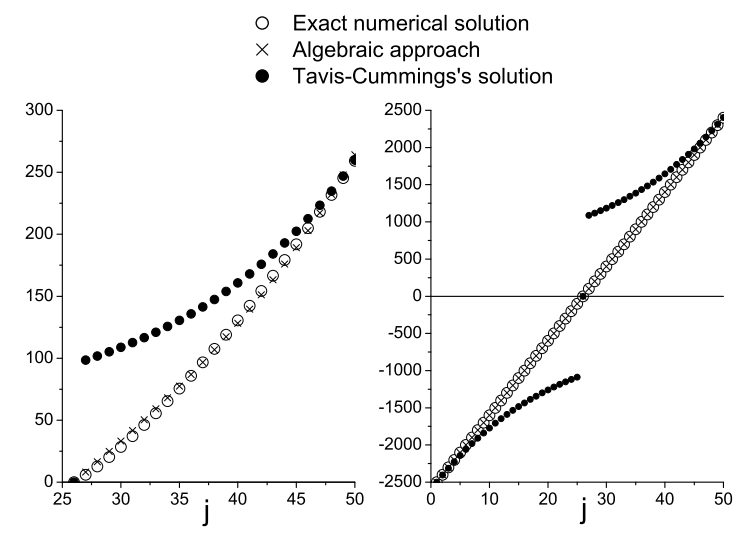

FIG. 4: Energy levels of $V$ in the ascending order for $r=25$, as calculated numerically (circles), from Eq. (38) (crosses), and as is given in [1] (squares). a) $M=50$; b) $M=2525$.

\section{shown in Fig. 3 .}

In their original article [1] Tavis and Cummings also found an approximative analytical expression for the spectrum of the interaction operator $V$. We compare the results of Ref. [1] with our analytical and numerical solutions in Fig. 6 . It is evident that the Tavis and Cummings solution is only accurate for very large and very small values of index $j$.

The Fig. 5 compares the energies calculated numerically and in accordance with the analytical solution Eq. (38). In the intermediate region of $M$ the curves for nearby and remote zones overlap and coincide thus providing still satisfactory correspondence to the exact solution. However evidently the expansion for the remote zone breaks down in the nearby zone and vice versa. This means that the classification of zones introduced in this paper is indeed adequate.

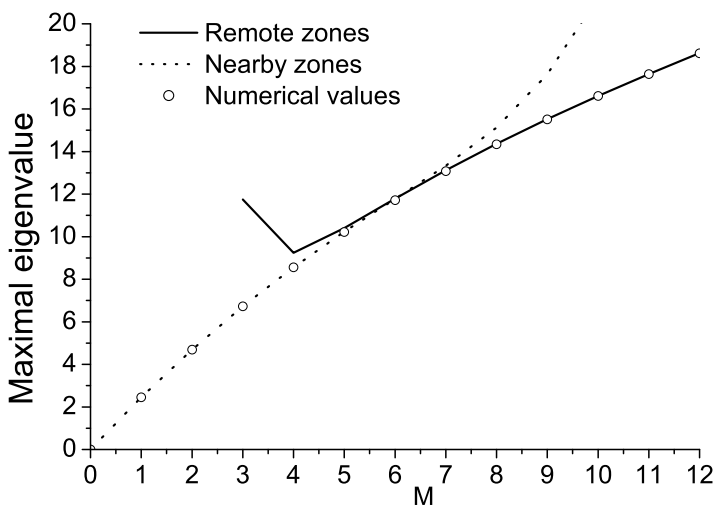

FIG. 5: The dependence on $M$ of the maximal eigenvalue of $V$ for $r=3$, as calculated numerically and from Eq.(38).

Finally we consider the $\mathcal{N}$-atom dressed states. In other words we introduce a representation in which the zero order Hamiltonian is diagonal. This representation is given by transformation $\tilde{U}_{0}$. For $\mathcal{N}=1$ all the higher order terms in Eq.(37) (higher than zero order) vanish, while the eigenstates of the zero order Hamiltonian coincide with the dressed states 41] of the Jaynes-Cummings model. We can thus call the eigenstates of the zero order Hamiltonian the dressed states of the $\mathcal{N}$-atom model. Notice also that in the remote zones the Rabi frequency Eq.(35) does not depend on the Dicke index $r$. If we consider only the zero order terms of $H$, it is convenient to combine all the remote zones that have the same Rabi frequency into a remote superzone, whose dimension is $2^{\mathcal{N}}$. Introducing an operator for the total number of quanta in the atom-field system (cf. the definition of $M$ in Eq.(25)),

$$
K=a^{\dagger} a+S_{3}+\frac{\mathcal{N}}{2}
$$

we can define the remote superzone as follows. A remote superzone contains all eigenvectors of $K$ that have the same eigenvalue, provided it is larger than $\mathcal{N}$. In Fig. 6 we show the definition of the zones in the TavisCummings model for $\mathcal{N}=4$.

It can be readily seen that, in the Hilbert subspace corresponding to the remote superzone, the unitary transformation $\tilde{U}_{0}$ can be factorized into a product of $\mathcal{N}$ singleparticle transformations that are the dressing operators for the Jaynes-Cummings problem (cf. Eq.(29)),

$$
\tilde{U}_{0}=\prod_{j=1}^{\mathcal{N}} \exp \left[\frac{\pi}{4}\left(\frac{1}{\sqrt{a^{\dagger} a+1}} a \sigma_{+}^{j}-\sigma_{-}^{j} a^{\dagger} \frac{1}{\sqrt{a^{\dagger} a+1}}\right)\right] .
$$

¿From the discussion following Eq.(29), it follows that in each remote superzone the field operators $\frac{1}{\sqrt{a^{\dagger} a+1}} a$ 


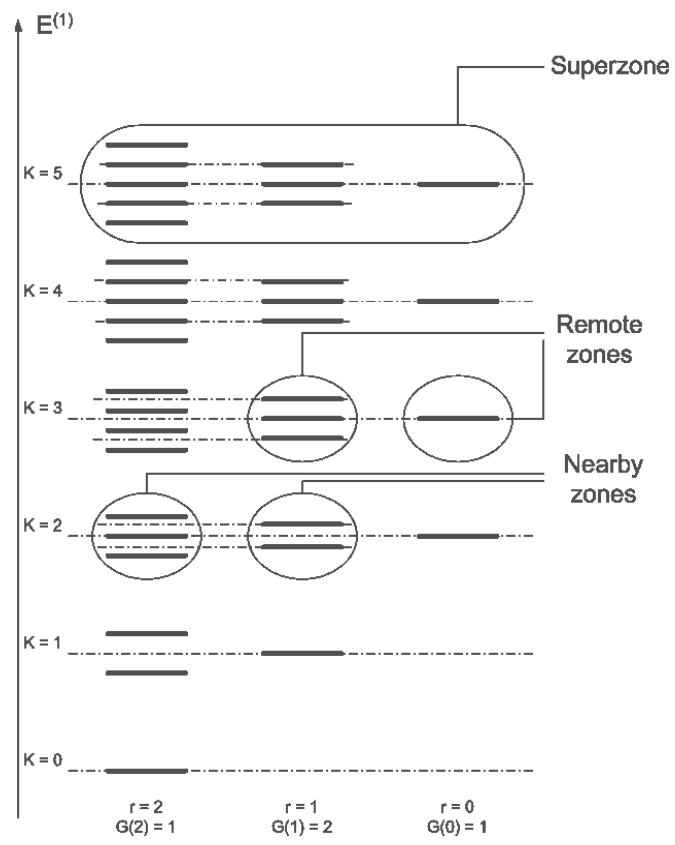

FIG. 6: Zones structure for $\mathcal{N}=4 . E^{(1)}$ is the spectrum of the zero order Hamiltonian. $K$ is an eigenvalue of the corresponding operator 39 ), and $G(r)$ is a number of equivalent irreps of $s u(2)$ defined below.

and $a^{\dagger} \frac{1}{\sqrt{a^{\dagger} a+1}}$ commute. This means that the system behaves almost like a semiclassical one.

\section{ENHANCEMENT OF SPONTANEOUS EMISSION IN THE RESONATOR DUE TO COLLECTIVE EFFECTS}

In the previous section we developed an algebraic approach to the Tavis-Cummings model. We introduced the operators $\tilde{S}_{ \pm}$describing collective excitations in the atom-field system. In terms of these operators we constructed a perturbation series for the Tavis-Cummings Hamiltonian Eq.(1). The derived perturbation series gives us a tool to distinguish and classify cooperative (multiparticle) effects of different orders that are involved in calculations of different physical observables characterizing the atom-field system. In the preceding section we constructed a Hamiltonian Eq.(37) corresponding to the zero order approximation for the Hamiltonian Eq.(11). This simplified operator depends on multiparticle Rabi frequency Eq. (35), which depends on the number of atoms in the cavity. Therefore the simplified Hamiltonian Eq.(37) allows to account for cooperative effects in the system.

In this section we study a contribution of cooperative effects into the rate of spontaneous emission generated by the atom-field system. The atom-field system is assumed to be prepared in the state of thermal equilibrium. This state is described by the canonical Gibbs ensemble with the thermostat temperature $T$. This means that under the "system" we imply $\mathcal{N}$ atoms strongly coupled to the resonator mode. Under the "thermostat" we imply the surrounding environment, for instance, cavity walls taken at the temperature $T$. In the state of thermal equilibrium the exact atom-field density matrix should be defined using the Hamiltonian Eq.(11). However, for the exact density matrix the analytical analysis of the transition probability, if possible at all, would be highly technically involved.

We demonstrate that nontrivial physical results for the intensity of spontaneous emission of $\mathcal{N}$ two-level atoms placed inside the cavity can be already obtained for the zero order approximation of the exact Hamiltonian Eq.(37). The thermal state is given by

$$
\rho_{t h}=\frac{1}{Z} \exp \left[-\frac{H_{0}+\frac{\Omega_{R}}{2}\left(\tilde{S}_{+}+\tilde{S}_{-}\right)}{k T}\right] .
$$

Here $Z$ is a normalization factor.

We show here that the intensity of spontaneous emission of the system comprised of $\mathcal{N}$ atoms strongly coupled to the resonator mode and prepared in the thermal state, can be greatly enhanced at a certain temperature. This amplification results from high correlations in the atomic subsystem. Similar effect exists for Dicke's superradiant state [2]. This state is prepared by a short laser pulse. Therefore the setting of the Dicke's theory is quite different from our considerations of the stationary state of thermal equilibrium.

When calculating the rate of spontaneous emission (or the intensity proportional to this quantity) we merely follow the ideas of Dicke's paper (see e.g. [2]). According to this theory, the rate of spontaneous emission in the system is proportional to the average of the square of the atomic dipole, viz.

$$
I=I_{0} \cdot\left\langle S_{+} S_{-}\right\rangle=I_{0} \cdot \operatorname{Tr}\left\{\rho_{t h} \cdot S_{+} S_{-}\right\} .
$$

It is convenient to calculate the average in the dressed states basis, where

$$
\rho_{t h} \rightarrow \frac{1}{Z} \tilde{U}_{0} \rho_{t h} \tilde{U}_{0}^{-1}=\exp \left[-\frac{H_{0}+\Omega_{R} \tilde{S}_{3}}{k T}\right] .
$$

Taking into account Eq.(14) along with the fact that, for an arbitrary zone,

$$
S_{3}=(\tilde{r}-r)+\tilde{S}_{3},
$$


it can be shown that in the dressed states basis

$$
\begin{gathered}
\tilde{U}_{0}\left(S_{+} S_{-}\right) \tilde{U}_{0}^{-1}=\tilde{r}\left(2 r-\frac{3}{2} \tilde{r}+\frac{1}{2}\right)- \\
\left(r+\frac{1}{2}-\tilde{r}\right)\left(\tilde{S}_{+} \tilde{S}_{-}\right)-\frac{1}{4}\left(\tilde{S}_{+}^{2}-2 \tilde{S}_{3}^{2}+\tilde{S}_{-}^{2}\right) .
\end{gathered}
$$

Finally, the intensity of spontaneous emission is given by

$$
\begin{gathered}
I=\frac{I_{0}}{Z} \sum_{M=0}^{\infty} \sum_{r=\varepsilon}^{\frac{\mathcal{N}}{2}} G(r) \\
\sum_{\tilde{m}=-\tilde{r}}^{\tilde{r}} e^{-\frac{\omega\left(M-r+\frac{\mathcal{N}}{2}\right)+\Omega_{R} \tilde{m}}{k T}}\left[\tilde{r}\left(2 r-\frac{3}{2} \tilde{r}+\frac{1}{2}\right)+\frac{1}{2} \tilde{m}^{2}\right] .
\end{gathered}
$$

Here $\varepsilon \equiv \frac{1-(-1)^{\mathcal{N}}}{4}$, and $G(r)=\frac{\mathcal{N} !(2 r+1)}{\left(\frac{N}{2}+r+1\right) !\left(\frac{N}{2}-r\right) !}$ is the number of equivalent representations with the same $r$.

Let us consider the intensity per atom, i.e. $I_{1} \equiv I / \mathcal{N}$. This intensity consists of two terms, i.e. the first is given by a single-particle contribution $I_{\text {single }}$ and the second one proportional to the two-particle correlation function Cor, $I_{1}=I_{\text {single }}+I_{0}(\mathcal{N}-1) C o r$. They are found to be

$$
\begin{gathered}
I_{\text {single }} \equiv I_{0} \frac{1}{\mathcal{N}}\left\langle\sum_{i} \sigma_{+}^{i} \sigma_{-}^{i}\right\rangle=I_{0}\left(\frac{1}{2}+\frac{1}{\mathcal{N}}\left\langle S_{3}\right\rangle\right), \\
C o r \equiv \frac{1}{\mathcal{N}(\mathcal{N}-1)}\left\langle\sum_{i \neq j} \sigma_{+}^{i} \sigma_{-}^{j}\right\rangle .
\end{gathered}
$$

It is plain that in the absence of the cavity, the correlation function vanishes and the only contribution to $I_{1}$ is given by the first term,

$$
I_{\text {single }}=I_{c l} \equiv I_{0}\left(1+e^{\frac{2 \omega}{k T}}\right)^{-1} .
$$

The contribution of any remote superzone can easily be found due to the factorization property of the operator Eq.(40). If we denote the trace over states that belong to the same remote superzone by subscript $K$, we obtain

$$
\begin{gathered}
\left\langle S_{+} S_{-}\right\rangle_{K}=\frac{\mathcal{N}}{2 Z} e^{\frac{K \omega}{k T}}\left(2 \cosh \left(\frac{\Omega_{R}}{2 k T}\right)\right)^{\mathcal{N}}+ \\
\frac{\mathcal{N}(\mathcal{N}-1)}{Z} e^{\frac{K \omega}{k T}}\left(2 \cosh \left(\frac{\Omega_{R}}{2 k T}\right)\right)^{\mathcal{N}-2}\left(\sinh \left(\frac{\Omega_{R}}{2 k T}\right)\right)^{2} .
\end{gathered}
$$

In Fig. 07 we compare the spontaneous emission $I_{1}$ in the presence of a cavity against the intensity $I_{c l}$ of $\mathcal{N}$ atoms in the absence of cavity.

Notice that if the number of atoms is big enough, the intensity of radiation exhibits a high maximum. In a cavity at low $T$, the cluster of $\mathcal{N}$ two-level atoms emits much more intensively than it does in the free space. It should be possible to drive the system to thermal equilibrium at the temperature where the spontaneous emission exhibits maximum. The marked amplification of spontaneous emission should be observed in cavity experiments.

Concluding this section, we recapitulate our main results. We consider spontaneous emission of the system comprised of $\mathcal{N}$ two-level atoms strongly coupled to the cavity mode and prepared in the state of thermal equilibrium. In the absence of the cavity the atoms in the thermal equilibrium would be uncorrelated. In this case

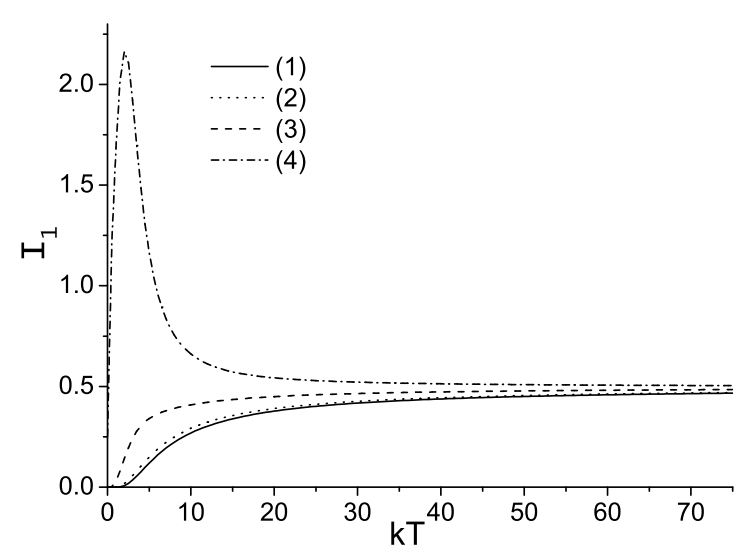

FIG. 7: The intensity of spontaneous emission per atom (in units of $I_{0}$ ) versus cavity temperature. The curve (1) is the classical result given by Eq.(48). The curves $(2,3,4)$ correspond to $\mathcal{N}=10, \mathcal{N}=50, \mathcal{N}=100$, respectively, and $\frac{\omega}{g}=10$.

the spontaneous emission would be described by the conventional formula Eq. 48). For high-Q resonators the strong coupling to the resonator mode should necessarily be taken into account. We demonstrate that in this case the intensity of spontaneous emission can be be greatly enhanced. This phenomenon can be explained by additional correlation between atoms established by the cavity mode. To analyze the effect analytically we have replaced the exact Tavis-Cummings Hamiltonian Eq.(1) by its zero order approximation derived in the previous sections. This allowed us to represent the intensity of spontaneous emission in the simple analytical form Eq.(46). It is appropriate to emphasize once again that the zero order approximation of the Hamiltonian contains strong coupling and, thus, describes cooperative effects in the atomic subsystem. This is the consequence of the fact that the operators $\tilde{S}_{ \pm}$describe collective excitations in the atom-field system.

\section{CONCLUSION}

In this work we solved the Tavis-Cummings problem by applying the technique of polynomially deformed algebras. We constructed the transformations that map one polynomial algebra of operators onto another. This allowed us to reformulate the problem in terms of a simpler algebra of second order, $\mathbb{S}_{r}$, and develop a specific perturbation theory. Our results have a significant advantage over the so-called linearization approximation, i.e. the case when the Hamiltonian is linearized in terms of the algebra $\mathfrak{U}_{1}$. In this latter approach a structure polynomial of a higher order is approximated by a polynomial of first order. This method allows only to calculate the lowest or highest eigenvalues, and the corresponding eigenstates. 
It does not take into account the finite dimensionality of the representation. The parabolic approximation developed in this work provides in this respect a significant advantage because it allows to construct a finite dimensional representation for the problem. We were able to find analytical expressions for all the eigenvalues of the Hamiltonian up to third order in the small parameter $\alpha$. For the nearby zones we showed explicitly how the collective quantum Rabi frequency depends on Dicke in$\operatorname{dex} r$. Since this index characterizes the symmetry of atomic states, the result has significant physical implications. The dependence on atomic symmetry is revealed already in zeroth order in the perturbation expansion. Employing our methods we found an interesting new effect, amplification of spontaneous emission of thermal $\mathcal{N}$ atom states due to collective effects. We expect that this phenomenon can be observed in cavity experiments. It is worth mentioning that the applicability of the method developed in this paper can be extended to many other problems, including Bose-Einstein condensation, multiphoton interactions in the micromaser, and multimode interaction of an electromagnetic field with matter. Our methods allow in particular to address the problem of collective and dressed states in the mentioned physical systems.

\section{ACKNOWLEDGEMENTS}

This work has been supported by the Academy of Finland (Project No. 44875).

I.V. is grateful to the Centre for International Mobility (CIMO) and University of Jyvaskyla for financial support.

$*$

\section{APPENDIX A: SIMILARITY TRANSFORMATIONS}

We look for similarity transformations $\tilde{U}_{k}$ that diagonalize the Hamiltonian in different orders of $\alpha$,

$$
V_{k}^{(n)} \equiv \tilde{U}_{k} V_{k-1}^{(n)} \tilde{U}_{k}^{-1},
$$

where $k=0,1,2,3$. In Eq. (A1)) only the terms of order $n$ in the small parameter $\alpha$ are present. For $k=0$, the term $V_{k=1}^{(n)}$ should be replaced by the corresponding term in Eq.(37). Regrouping the terms we obtain

$$
\mathrm{V}^{(0)}=2 \tilde{S}_{x}\left(1-\frac{1}{2}\left(\frac{\alpha}{4}\right)^{2}\right),
$$

$$
\begin{gathered}
\mathrm{V}^{(1)}=-\frac{\alpha}{2}\left(1+\frac{1}{4}\left(\frac{\alpha}{4}\right)^{2}\right) \cdot B \\
\mathrm{~V}^{(2)}=-\left(\frac{\alpha}{2}\right)^{2} \cdot\left[\tilde{S}_{3} \frac{\tilde{S}_{+}+\tilde{S}_{-}}{2} \tilde{S}_{3}\right] \\
\mathrm{V}^{(3)}=-\frac{1}{2}\left(\frac{\alpha}{2}\right)^{3}\left[\tilde{S}_{3} \cdot B \cdot \tilde{S}_{3}\right]
\end{gathered}
$$

where

$$
B \equiv\left[\tilde{S}_{3} \tilde{S}_{x}+\tilde{S}_{x} \tilde{S}_{3}\right]
$$

and

$$
\tilde{S}_{x} \equiv \frac{\tilde{S}_{+}+\tilde{S}_{-}}{2} .
$$

\section{The zero order transformation $\tilde{U}_{0}$}

As known from the theory of $s u(2)$ algebra, the operator $\tilde{S}_{x}$ can be diagonalized by the transformation

$$
\tilde{U}_{0}=\exp \left[\frac{\pi}{4}\left(\tilde{S}_{+}-\tilde{S}_{-}\right)\right]=\exp \left[-i \frac{\pi}{2} \tilde{S}_{y}\right]
$$

Employing this transformation we obtain

$$
\begin{array}{r}
\mathrm{V}_{0}^{(0)}=2 \tilde{S}_{3}\left(1-\frac{1}{2}\left(\frac{\alpha}{4}\right)^{2}\right), \mathrm{V}_{0}^{(1)}=-\mathrm{V}^{(1)} \\
\mathrm{V}_{0}^{(2)}=-\left(\frac{\alpha}{2}\right)^{2} \cdot\left[\tilde{S}_{x} \tilde{S}_{3} \tilde{S}_{x}\right] \\
\mathrm{V}_{0}^{(3)}=\frac{1}{2}\left(\frac{\alpha}{2}\right)^{3}\left[\tilde{S}_{x} B \tilde{S}_{x}\right] .
\end{array}
$$

\section{The first order transformation $\tilde{U}_{1}$}

It can readily be seen that the transformation

$$
\tilde{U}_{1}=\exp [\alpha x \cdot D 1], \quad D 1 \equiv-i\left[\tilde{S}_{3} \tilde{S}_{y}+\tilde{S}_{y} \tilde{S}_{3}\right]
$$

diagonalizes the operators in the first order. In the diagonalization one needs the commutators 


$$
\begin{array}{r}
{\left[\tilde{S}_{3}, D 1\right]=B,} \\
{\left[\left[\tilde{S}_{3}, D 1\right], D 1\right]=4 \tilde{S}_{3}\left(\tilde{S}^{2}-2 \tilde{S}_{3}^{2}-\frac{1}{4}\right),} \\
{\left[\left[\left[\tilde{S}_{3}, D 1\right], D 1\right], D 1\right]=\left(4 \tilde{S}^{2}-1\right) B-8\left[\tilde{S}_{3} B+B \tilde{S}_{3}-\tilde{S}_{3} \tilde{S}_{x} \tilde{S}_{3}\right],} \\
{\left[\tilde{S}_{3} \tilde{S}_{x} \tilde{S}_{3}, D 1\right]=2 \tilde{S}_{3}^{2}\left(\tilde{S}^{2}-2 \tilde{S}_{3}^{2}\right)+\left(\tilde{S}_{3} \tilde{S}_{x}\right)^{2}+\left(\tilde{S}_{x} \tilde{S}_{3}\right)^{2}}
\end{array}
$$

Then up to third order in $\alpha$,

$$
\begin{array}{r}
\tilde{U}_{1} \mathrm{~V}_{0}^{(0)}\left(\tilde{U}_{1}\right)^{-1}=\mathrm{V}_{0}^{(0)}-2 \alpha x\left(1-\frac{1}{2}\left(\frac{\alpha}{4}\right)^{2}\right) B+ \\
4(\alpha x)^{2} \tilde{S}_{3}\left(\tilde{S}^{2}-2 \tilde{S}_{3}^{2}-\frac{1}{4}\right)-\frac{(\alpha x)^{3}}{3} \\
\left\{\left(4 \tilde{S}^{2}-1\right) B-8 \tilde{J}\right\}
\end{array}
$$

and

$$
\begin{gathered}
\tilde{U}_{1} \mathrm{~V}_{0}^{(1)}\left(\tilde{U}_{1}\right)^{-1}=\frac{\alpha}{2}\left(1+\frac{1}{4}\left(\frac{\alpha}{4}\right)^{2}\right) \cdot B- \\
\alpha^{2} x \cdot 2 \tilde{S}_{3}\left(\tilde{S}^{2}-2 \tilde{S}_{3}^{2}-\frac{1}{4}\right)+\alpha^{3}\left(\frac{x}{2}\right)^{2} . \\
\left\{\left(4 \tilde{S}^{2}-1\right) B-8 \tilde{J}\right\},
\end{gathered}
$$

where $\tilde{J} \equiv \tilde{S}_{3} B+B \tilde{S}_{3}-\tilde{S}_{3} \tilde{S}_{x} \tilde{S}_{3}$.

$$
\begin{gathered}
\tilde{U}_{1} \mathrm{~V}_{0}^{(2)}\left(\tilde{U}_{1}\right)^{-1}=\mathrm{V}_{0}^{(2)}+ \\
\alpha^{3}\left(\frac{x}{2}\right)^{2} \cdot\left\{\tilde{S}_{x} \tilde{S}_{3}\left(2 \tilde{S}^{2}-4 \tilde{S}_{3}^{2}-\tilde{S}_{x}^{2}\right)+\left(2 \tilde{S}^{2}-4 \tilde{S}_{3}^{2}-\tilde{S}_{x}^{2}\right) \tilde{S}_{3} \tilde{S}_{x}\right\}
\end{gathered}
$$

In third order the operator $V_{0}^{(3)}$ remains unchanged after and find then that the transformation, i.e. $V_{1}^{(3)}=V_{0}^{(3)}$. In order to calculate $V_{0}^{(2)}$ up to third order, we take into account that

$$
x=\frac{1}{4} \frac{\left(1+\left(\frac{\alpha}{8}\right)^{2}\right)}{\left(1-2\left(\frac{\alpha}{8}\right)^{2}\right)} \approx \frac{1}{4} \cdot\left(1+3\left(\frac{\alpha}{8}\right)^{2}\right),
$$

$$
\begin{gathered}
\mathrm{V}_{1}^{(0)}=\mathrm{V}_{0}^{(0)}-4\left(\frac{\alpha}{4}\right)^{2} \tilde{S}_{3}\left(\tilde{S}^{2}-2 \tilde{S}_{3}^{2}-\frac{1}{4}\right), \quad \mathrm{V}_{1}^{(1)}=0, \quad \mathrm{~V}_{1}^{(2)}=\mathrm{V}_{0}^{(2)}, \\
\mathrm{V}_{1}^{(3)}=\mathrm{V}_{0}^{(3)}-\frac{1}{2}\left(\frac{\alpha}{4}\right)^{3} B+\frac{2}{3}\left(\frac{\alpha}{4}\right)^{3}\left\{\left(4 \tilde{S}^{2}-1\right) \cdot B-8 \tilde{J}\right\}+ \\
\left(\frac{\alpha}{4}\right)^{3} \cdot\left\{\tilde{S}_{x} \tilde{S}_{3}\left(2 \tilde{S}^{2}-4 \tilde{S}_{3}^{2}-\left(\tilde{S}_{x}\right)^{2}\right)+\left(2 \tilde{S}^{2}-4 \tilde{S}_{3}^{2}-\left(\tilde{S}_{x}\right)^{2}\right) \tilde{S}_{3} \tilde{S}_{x}\right\} .
\end{gathered}
$$

\section{The second and the third orders transformations}

To find the second order transformation we rewrite $\mathrm{V}_{1}^{(2)}$ in a symmetrized form,

$$
\mathrm{V}_{1}^{(2)}=-\frac{1}{2}\left(\frac{\alpha}{2}\right)^{2}\left[\tilde{L}_{x} \tilde{S}_{3}+\tilde{S}_{3} \tilde{L}_{x}+\tilde{S}_{3}\left(\tilde{S}^{2}-\tilde{S}_{3}^{2}-1\right)\right]
$$


where $\tilde{L}_{x} \equiv \frac{\tilde{S}_{+}^{2}+\tilde{S}_{-}^{2}}{4}$. The diagonalizing transformation is then given by

$$
\tilde{U}_{2} \equiv \exp \left\{i \frac{1}{2}\left(\frac{\alpha}{4}\right)^{2} \cdot\left[\tilde{L}_{y} \tilde{S}_{3}+\tilde{S}_{3} \tilde{L}_{y}\right]\right\},
$$

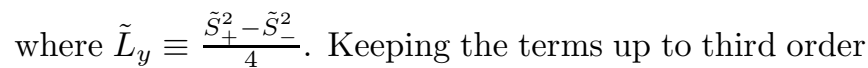
we obtain (see (A13))

$$
\tilde{U}_{2} \mathrm{~V}_{1}^{(0)}\left(\tilde{U}_{2}\right)^{-1}=\mathrm{V}_{1}^{(0)}+\frac{1}{2}\left(\frac{\alpha}{2}\right)^{2}\left[\tilde{L}_{x} \tilde{S}_{3}+\tilde{S}_{3} \tilde{L}_{x}\right](. \mathrm{A} 18
$$

The transformation A17 does not change the expressions, given above for $V_{1}^{(2)}$ and $V_{1}^{(3)}$, and we find that

$$
\begin{gathered}
\mathrm{V}_{2}^{(0)}=\mathrm{V}_{1}^{(0)}-\left(\frac{\alpha}{4}\right)^{2} \cdot 2 \tilde{S}_{3}\left(\tilde{S}^{2}-\tilde{S}_{3}^{2}-1\right) \\
\mathrm{V}_{2}^{(1)}=\mathrm{V}_{2}^{(2)}=0, \quad \mathrm{~V}_{2}^{(3)}=\mathrm{V}_{1}^{(3)} .
\end{gathered}
$$

Diagonalization of $V_{2}^{(3)}$ can be performed in a similar way with an operator $\tilde{U}_{3}=\exp \left[-\left(\frac{\alpha}{4}\right)^{3} \cdot \mathcal{O}\right]$. As there are no diagonal terms in $V_{2}^{(3)}$, which would contribute to the spectrum of the Hamiltonian, we do not give here the fairly complicated form of operator $\mathcal{O}$. The final diagonal form for the interaction $V$ is thus given by

$$
\bar{V}=\Omega \tilde{S}_{3}\left\{1+\left(\frac{\alpha}{4}\right)^{2}\left[5 \tilde{S}_{3}^{2}-3 \tilde{r}(\tilde{r}+1)+1\right]\right\} .
$$

To recapitulate, we introduced four transformations $\tilde{U}_{k}, \quad k=0,1,2,3$, which successively diagonalize the interaction operator in the Tavis-Cummings Hamiltonian up to third order with respect to the small parameter $\alpha=\left(\frac{1}{2} \Omega_{R}\right)^{-2}$, with $\Omega_{R}$ the generalized Rabi frequency of Eq.(35), such that

$$
\bar{V} \equiv \tilde{U} V \tilde{U}^{-1}, \quad \tilde{U} \equiv \tilde{U}_{3} \tilde{U}_{2} \tilde{U}_{1} \tilde{U}_{0}
$$

[1] M. Tavis and F. W. Cummings, Phys. Rev. 170, 379 (1968).

[2] R. Dicke, Phys. Rev. 93, 99 (1954).

[3] E. Jaynes and F. Cummings, Proc. IEEE 51, 89 (1963).

[4] D. Meschede, H. Walther, and G. Muller, Phys. Rev. Lett. 54, 551 (1985).

[5] P. Filipowicz, J. Javanainen, and P. Meystre, Phys. Rev. A 34, 3077 (1986).

[6] P. Elmfors, B. Lautrup, and B.-S. Skagerstam, Phys. Rev. A 54, 5171 (1996).

[7] J.M. Raimond, M. Brune, and S. Haroche, Rev. Mod. Phys. 73, 565 (2001).

[8] S. Yu, H. Rauch, and Y. Zhang, Phys. Rev. A 52, 2585 (1995).

[9] X. Yang, Y. Wu, and Y. Li, Phys. Rev. A 55, 4545 (1997).

[10] A. Rybin, G. Kastelewicz, J. Timonen, and N. Bogoliubov, J. Phys. A 31, 4705 (1998); N.M. Bogoliubov, R.K. Bullough, and J. Timonen, J. Phys. A 29, 6305 (1996);

[11] S. Carusotto, Phys. Rev. A 40, 1848 (1989).

[12] M.E. Smithers, and E.Y.C. Lu, Phys. Rev. A 9, 790 (1974).

[13] B.R. Mollow, and R.J. Glauber, Phys. Rev. 160, 1076 (1967).

[14] J. Tucker, and D.F. Walls, Phys. Rev. 178, 2036 (1969).

[15] A.B. Klimov, and L.L. Sanchez-Soto, Phys. Rev. A 61, $063802(2000)$.

[16] M. Kozierowski, and A.A. Mamedov, and S.M. Chumakov, Phys. Rev. A 42, 1762 (1990).

[17] S.M. Chumakov, and A.B. Klimov, and J.J. SanchezMondragon, Phys. Rev. A 49, 4972 (1994).

[18] C. Saavedra, and A.B. Klimov, and S.M. Chumakov, and J.C. Retamal, Phys. Rev. A 58, 4078 (1998).

[19] J. Delgado, and E.C. Yustas, and L.L. Sanchez-Soto, A.B. Klimov, and Phys. Rev. A 63, 063801 (2001).

[20] C. Brif, Phys. Rev. A 54, 5253 (1996).
[21] V. Sunilkumar, B.A. Bambah, R. Jagannathan, P.K. Panigrahi, andV. Srinivasan, J.Opt.B 2, 126 (2000).

[22] T. Holstein and H. Primakoff, Phys. Rev. 58, 1098 (1940).

[23] F. Persico, and G. Vetri, Phys. Rev. A 12, 2083 (1975).

[24] D. Bonatsos, C. Daskaloyannis, P. Kolokotronis, J. Phys. A 26, L871 (1993).

[25] D. Ruan, Y. Jia, and H. Sun, quant-ph/0111007.

[26] V.P. Karassiov, J. Phys. A 27, 153 (1994); V.P. Karassiov, A.A. Gusev, and S.I. Vinitsky, quantph/0112040(2001).

[27] D. Bonatsos, C. Daskaloyannis, G. A. Lalazissis, Phys. Rev. A 47, 3448 (1993).

[28] R. Brandt and O. W. Greenberg, J. Math. Phys. 10, 1168 (1969).

[29] M. Rasetti, Int. J. Theor. Phys. 5, 377 (1972).

[30] J. Katriel, Phys. Lett. 72A, 94 (1979).

[31] C. Gerry, J. Phys. A 16, 313 (1971).

[32] J. Katriel, A. I. Solomon, G. DAriano, and M. Rasetti, Phys. Rev. D 34, 2332 (1986).

[33] S. Carusotto, Phys. Rev. A 38, 3249(1971).

[34] D. Bonatsos, C. Daskaloyannis, P. Kolokotronis, and D. Lenis, hcp-th/9402099.

[35] P. Shanta, S. Chaturvedi, V. Srinivasan, G.S. Agarwal, and C.L. Mehta, Phys. Rev. Lett. 72, 1447(1994).

[36] R. Floreanini, L. Lapointe, L. Vinet, Phys. Lett. B 389, 327(1996).

[37] D. Ruan, W. Ruan, Phys. Lett. A 263, 78(1999).

[38] R.J. Glauber, Phys. Rev. 131, 2766(1963).

[39] A. Rybin, G.P. Miroshnichenko, I.P. Vadeiko, and J. Timonen, J. Phys. A 32, 8739 (1999).

[40] W. Witschel, J. Phys. A 7, 1847 (1974).

[41] M.O. Scully and M.S. Zubairy, Quantum Optics, Cambridge University Press, 1997. 